\title{
Towards Provenance Integration for Field Devices in Industrial IoT Systems
}

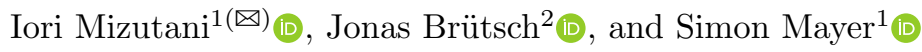 \\ 1 University of St.Gallen, St.Gallen, Switzerland \\ \{iori.mizutani, simon.mayer\}@unisg.ch \\ 2 ETH Zürich, Zürich, Switzerland \\ brujonas@ethz.ch \\ https://interactions.ics.unisg.ch/
}

\begin{abstract}
Industrial Internet of Things (IoT) systems are becoming increasingly complex with interconnected field devices. To cope with the complexity and to facilitate further interoperability between heterogeneous field devices, it is necessary to have a holistic understanding of different types of provenance information about field devices and associated processes. In this paper, we investigate the potential integration of such provenance with the W3C Web of Things and current industrial standards to realize more explainable, efficient, and safer industrial IoT systems.
\end{abstract}

Keywords: Web of Things $\cdot$ Industrial IoT $\cdot$ OPC UA $\cdot$ Thing description $\cdot$ Semantic web $\cdot$ Provenance

\section{Introduction}

The Internet of Things (IoT) is a key enabler for the integration of control and automation processes in a wide variety of industrial sectors adding flexibility, robustness, and responsiveness to industrial automation systems [7]. A principle called "separation of concerns" draws the boundary between the information technology (IT) and the operational technology (OT) allowing functional modularity by isolating responsibility. However, with increased connectivity among heterogeneous field devices and software components in complex (industrial) IoT systems, convergence of IT and OT is required to orchestrate the whole automation execution. For instance, to examine to what extent a particular manufacturing process increases the degradation of a field device and its maintenance cost, a factory manager would need the following provenance information: Where is the yield report for the process located?; What control logic was used?; Which field devices are involved?; and What is the context of these devices, and how do they relate to one another on the shop floor?. Since these questions stretch across different levels of abstraction and also conflate IT and OT concerns, it is necessary to have a holistic understanding of industrial IoT systems, which, importantly, includes different types of provenance information about field devices and associated processes in the automation system.

(C) Springer Nature Switzerland AG 2021

B. Glavic et al. (Eds.): IPAW 2020/IPAW 2021, LNCS 12839, pp. 250-255, 2021.

https://doi.org/10.1007/978-3-030-80960-7_21 


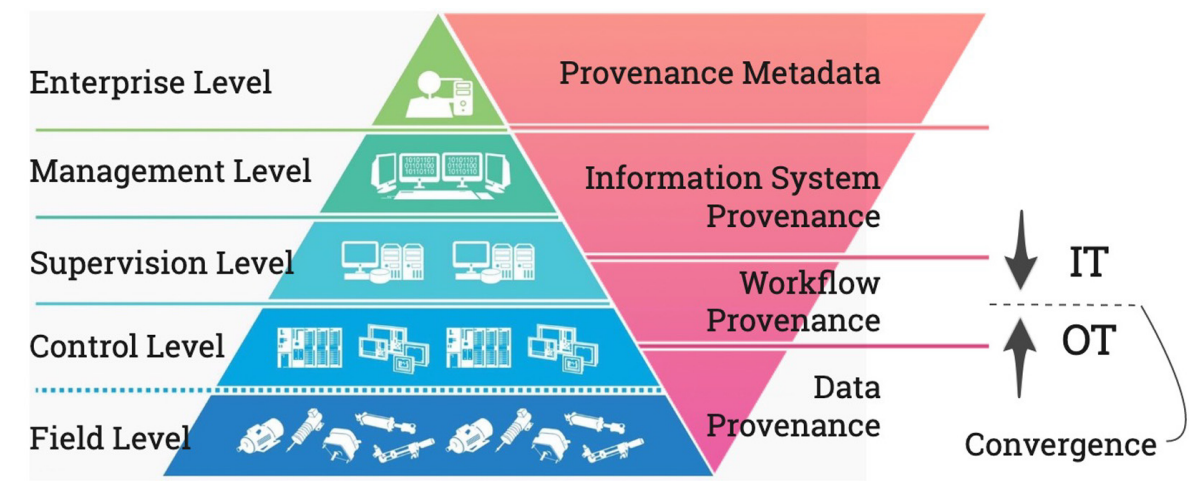

Fig. 1. Relationship between the types of provenance proposed in [3] with the layers of the automation pyramid.

In Fig. 1, we show the well-known ISA-95 automation pyramid [1] for industrial automation systems, where the upper three layers are dominated by IT concerns and the lower two are dominated by OT concerns. We observe that different types of provenance are generated on different layers of the automation pyramid, and propose a mapping of these layers to the provenance types proposed by [3]. Based on this observation, we propose to integrate provenance information across the automation pyramid by linking it to current research and standardization efforts in the Web of Things (WoT) domain. Concretely, we claim that the W3C WoT Architecture [6] is useful to overcome this separation with its platform-neutral environment to integrate cross-vendor and crossdomain IoT applications. In the W3C WoT Architecture, descriptive metadata for IoT devices and services is serialized into machine-understandable Thing Descriptions (TDs) [5] that allow clients to interact with heterogeneous thingprovided services by providing metadata about interactions, including protocol bindings, based on a vocabulary.

\section{Provenance and Field Devices}

In the following, we address four types of provenance information that are defined in [3] and adapt these for field devices based on the W3C WoT Architecture: (i.) provenance metadata (metadata describing an arbitrary derivation process for provenance information), (ii.) information system provenance (information pipeline that enables capturing and dissemination of information in the IT layers), (iii.) workflow provenance (provenance about the workflow in the OT layers), and ( $i v$.) data provenance (provenance information of events to/from field devices). 


\subsection{Provenance Metadata}

Provenance metadata is the most general type of information that encompasses the derivation process for any possible provenance information related to a field device. To describe an arbitrary provenance information derivation process for a field device, Interaction Affordances of TD can be utilized. The Interaction Affordances suggests a WoT client how it can interact with event, function, and state of the thing with three sub-types: EventAffordance, ActionAffordance, and PropertyAffordance, respectively. The TD context can be extended with semantic annotations with vocabularies defined in industrial knowledge bases, and a rich set of Semantic Web tooling allows to adapt an arbitrary data model for provenance data. In particular, a knowledge base such as the SOSA ontology [4] can be used to align the data provenance from field devices with other ontologies (e.g., W3C PROV-O), and to elicit implicitly hidden domain knowledge on the provenance information derived from field devices, which can then be shared among industrial IoT applications at different layers. Meanwhile, computing such provenance in a real-time context requires a seamless information exchange between field devices and applications.

\subsection{Information System Provenance}

Information system provenance refers to metadata about informationdisseminating processes within an information system, and such provenance usually belongs to the IT layer. As described in Fig. 1, such informationdisseminating processes can be observed between different levels in industrial automation systems. For example, a Supervisory Control and Data Acquisition (SCADA) system at the supervision level monitors and aggregates the information collected from the control level underneath. The SCADA system then exchanges the information with a Manufacturing Execution System (MES) at the management level to provision the underlying automation systems and also to coordinate the manufacturing process with the business planning, such as an alignment with the demanded Key Performance Indicator (KPI). To facilitate the information exchange in industrial IoT systems, the Open Platform Communications (OPC) foundation defines the OPC Unified Architecture (OPC UA) specification [8] as a communication framework for automation components.

OPC UA is used for an information system to aggregate complex information as Objects in an address space which can be accessed with defined OPC UA Services [9] . The interactions between the OPC UA Services could be exploited for constructing the information system provenance by collecting inputs, outputs, and configurations of the services. However, the OPC UA Service definitions are abstract descriptions and do not represent a specification for implementation. This opaqueness of the OPC UA Services makes it difficult to investigate internal information processing, which may degrade the quality of the information system provenance. In other words, the information system provenance can be observed within the OPC UA silo, but transitive provenance discovery into the OPC UA 
Services may be blocked. Consequently, this would decrease the transparency during the information disseminating process capturing.

To improve the transparency, we need to describe and expose the internal logic of the OPC UA Services for external IoT applications, such as a WoT client. Nevertheless, whether or not this can be achieved depends on how the service is actually implemented, i.e., in what programming language, in what framework, and on what platform. Hence, the investigation of the OPC UA Service implementation is required while further specification may be desirable.

\subsection{Workflow Provenance}

Whereas the information system provenance resides mostly in the IT layers, workflow provenance predominantly involves information in the OT layers. For the development, operation, and maintenance of an automation system, field devices are usually first configured "offline" and the logical configurations will be validated against their physical representative in a real deployment, such as their form factors and (spatial) locations. These properties are individual to each instance of field devices and are essential for the field-level elucidation in tracing the physical source of data, e.g., where and how a device is installed including the physical wiring topology and spatial constraints. Such descriptions are referred to as offline engineering information, and they are often represented in various data formats, e.g., Computer-aided Design (CAD) models, electrical and mechanical planning, Programmable Logic Controller (PLC) programming, and models of the human-machine interface. These formats and information models are defined and maintained by a number of vendors and domain standardization groups $^{1}$, and the OPC UA Companion Standards extend the OPC UA Device Information Model (DI) [10] for enhanced interoperability among different industrial domains.

Once the offline engineering is completed, field devices establish communications with other automation components in the system. The OPC Field Level Communication (FLC) initiative ${ }^{2}$ has been recently established to standardize the semantics and behaviors of field devices and controllers from different manufacturers. The OPC FLC is planning to extend the existing DI model for the management of different types of communication modes (e.g., Client/Server, PubSub) conforming within the required QoS. However, since it is extending the DI model, that information also needs to be accessed through the OPC UA Services. To semantify the field level communications for workflow provenance, the TD can also be extended to the future FLC specification. In the WoT architecture, Protocol Bindings define the mapping between affordance and concrete protocol message. Although, only an HTTP binding is currently standardized [5], further development is expected to support field level industrial protocols such as OPC UA and NETCONF for Time-Sensitive Networking (TSN) [13].

\footnotetext{
1 https://opcfoundation.org/markets-collaboration/.

${ }^{2}$ https://opcfoundation.org/flc/.
} 
For example, the control logic (e.g., machining of a metal rod by a turning machine) is written as a program inside the PLC, and can be described in Automation Markup Language ${ }^{3}$ with the companion specification for the OPC UA object model, and an OPC client application is able to query the control logic as workflow provenance. The engineering of the control logic can also be translated into a formalism (e.g., a UML state machine). This formalism then can be represented by Semantic Web technology, such as Web Ontology Language (OWL), and reasoning on the ontology realizes automated formal verification of the control logic for safety and reliability of the system [12]. In the verification process, the TD for field devices can be treated as an entity in the OWL ontology. Therefore, integration of the OPC DI model and the TD would enhance the interoperability between field level automation components (i.e., field devices) in the OT layers and the industrial IoT applications in the IT layers.

\subsection{Data Provenance}

In order to complete a specific task, a controller may provide commands and set point information to a field device, which then reports feedback after completion, or even coordinates further activities with other devices. Current industry trends expect more and more field devices to provide a rich set of information, and IT layer applications to extract and derive meaning from the data directly, without needing to go through the controllers.

From the viewpoint of the shop floor, every field device is a functional entity that consumes input data and produces output data. Instances of such data inputs and outputs are handled as events in industrial IoT systems. These events to/from field devices represent specific transient occurrences, such as device configuration changes, operational instructions, or individual sensor readings. They can also be instantiated as Object instances in OPC UA with the OPC UA DI model. A separate specification called Historical Data Access provides access to the historical values in Object instances, and allows the user to query the data within a specified time range. However, in a practical scenario, it is more convenient to store and access these data in a well-known storage, but this also requires translation of (tabular) data from databases into meaningful objectproperty forms in OPC UA [2].

To facilitate access to such events to/from field devices for the industrial IoT applications (including those non-compliant with OPC UA), we propose that EventAffordances of the W3C WoT TD could be utilized to capture the events in the OPC UA systems, and to allow WoT clients to directly interact with data stores that are external to the OPC UA. The TD would also allow subscription to events and retrieval of data for Web-based IoT applications while maintaining compatibility with the DI model, using a formal mapping between OPC UA and the Semantic Web [11], for example.

\footnotetext{
${ }^{3}$ https://www.automationml.org/.
} 


\section{Conclusion and Outlook}

Concepts from research on the Web of Things and from current standardization efforts within the W3C Web of Things have the potential to support the integration of provenance information across different layers of industrial IoT systems. Such integration would promote the convergence of IT and OT so that we can create more intelligent industrial IoT applications, which are not restricted by the IT/OT boundary. We believe that an important step is the semantic interoperability for existing technologies and standards in the industrial IoT. With respect to the integration of provenance information, this has the potential to make complex industrial IoT systems more explainable, efficient, and also safer.

\section{References}

1. ANSI: ANSI/ISA 95.00.01-2010 (IEC 62264-1 Mod). Standard, ANSI, May 2010

2. El Kaed, C., Ponnouradjane, A.: A model driven approach accelerating ontologybased IoT applications development. In: SEMANTICS Workshops (2017)

3. Herschel, M., Diestelkämper, R., Ben Lahmar, H.: A survey on provenance: what for? what form? what from? VLDB J. 26(6), 881-906 (2017). https://doi.org/10. 1007/s00778-017-0486-1

4. Janowicz, K., Haller, A., Cox, S.J., Le Phuoc, D., Lefrançois, M.: SOSA: a lightweight ontology for sensors, observations, samples, and actuators. J. Web Semant. 56, 1-10 (2019). https://doi.org/10.1016/j.websem.2018.06.003

5. Kamiya, T., McCool, M., Käbisch, S., Kovatsch, M., Charpenay, V.: Web of Things (WoT) Thing Description. Recommendation, W3C, April 2020. https://www.w3. org/TR/2020/REC-wot-thing-description-20200409/

6. Kovatsch, M., Matsukura, R., Lagally, M., Kawaguchi, T., Toumura, K., Kajimoto, K.: Web of Things (WoT) Architecture. Recommendation, W3C (2020). https:// www.w3.org/TR/wot-architecture/

7. Lee, J., Bagheri, B., Kao, H.A.: A cyber-physical systems architecture for industry 4.0-based manufacturing systems. Manufact. Lett. 3, 18-23 (2015). https://doi. org/10.1016/j.mfglet.2014.12.001

8. OPC Foundation: OPC 10000-1: OPC Unified Architecture - Part 1: Overview and Concepts. Specification, OPC Foundation (2017)

9. OPC Foundation: OPC 10000-4: OPC Unified Architecture - Part 4: Services. Specification, OPC Foundation (2017)

10. OPC Foundation: OPC 10000-100 - Part 100: Device Information Model. Specification, OPC Foundation (2021)

11. Schiekofer, R., Grimm, S., Brandt, M.M., Weyrich, M.: A formal mapping between OPC UA and the semantic web. In: 2019 IEEE 17th International Conference on Industrial Informatics (INDIN), vol. 1, pp. 33-40 (2019). https://doi.org/10.1109/ INDIN41052.2019.8972102

12. Schneider, G.F., Wicaksono, H., Ovtcharova, J.: Virtual engineering of cyberphysical automation systems: the case of control logic. Adv. Eng. Inform. 39, 127-143 (2019). https://doi.org/10.1016/j.aei.2018.11.009

13. Sciullo, L., Bhattacharjee, S., Kovatsch, M.: Bringing deterministic industrial networking to the W3C web of things with TSN and OPC UA. In: Proceedings of the 10th International Conference on the Internet of Things. pp. 1-8 (2020). https:// doi.org/10.1145/3410992.3410997 